\title{
Comparative analysis of cluster versus conventional immunotherapy in patients with allergic rhinitis
}

\author{
QIJUN FAN ${ }^{1}$, XUEJUN LIU $^{2}$, JINJIAN GAO $^{2}$, SAIYU HUANG $^{2}$ and LIYAN NI ${ }^{1}$ \\ ${ }^{1}$ Desensitization Treatment Center in the Department of Otolaryngology; ${ }^{2}$ Department of Otorhinolaryngology, \\ The Second Affiliated Hospital and Yuying Children's Hospital of Wenzhou Medical University, \\ Wenzhou, Zhejiang 325009, P.R. China
}

Received May 12, 2015; Accepted May 24, 2016

DOI: $10.3892 / \mathrm{etm} .2017 .4032$

\begin{abstract}
The present study aimed to compare the efficacy and safety of subcutaneous immunotherapy with Dermatophagoides pteronyssinus standardized extract administered in conventional and cluster immunotherapy regiments for patients with persistent allergic rhinitis. A total of 60 patients with moderate to severe allergic rhinitis caused by dust mites were treated for 1 year with either conventional immunotherapy $(n=30)$ or cluster immunotherapy $(n=30)$. Nasal conjunctival symptoms and signs were assessed to evaluate the clinical efficacy of the two regimens, and the incidence of local and systemic adverse reactions were also evaluated. The findings demonstrated that the cluster regimen reduced the duration between the initial and maintenance dose by $>60 \%$, and resulted in a significant improvement, as compared with the conventional regimen, after 6 weeks of observation $(\mathrm{P}<0.05)$. However, the incidence of local and systemic adverse reactions in the cluster regimen during the dose accumulation phase and the dose maintenance phase was not significantly different, as compared with the conventional immunotherapy regimen. These results suggest that cluster immunotherapy is efficacious and safe to treat patients who are clinically sensitive to dust mites.
\end{abstract}

\section{Introduction}

Allergic rhinitis (AR) is an allergic disease mediated by $\mathrm{IgE}$, which is increasing in incidence. The important role of specific immunotherapy (SIT) and its mechanism, safety and effectiveness against disease progression have become better

Correspondence to: Dr Liyan Ni, Desensitization Treatment Center in the Department of Otolaryngology, The Second Affiliated Hospital and Yuying Children's Hospital of Wenzhou Medical University, 109 Xueyuan Road, Wenzhou, Zhejiang 325009, P.R. China

E-mail: niliyan2001@163.com

Key words: allergic rhinitis, cluster immunotherapy, conventional immunotherapy, clinical efficacy, safety understood in recent years. The main advantage of SIT is its ability to alleviate clinical symptoms and reduce a patient's dependence on allergy medication. SIT is the only therapy that can alter the natural course of allergic diseases through an immunoregulatory mechanism (1). In 2008, the World Health Organization's Allergic Rhinitis and its Impact on Asthma Workshop Group indicated that specific immunotherapy is used as the main therapy to treat $\operatorname{AR}(2,3)$. The usual course of therapy with SIT includes subcutaneous immunotherapy (SCIT) and sublingual immunotherapy. SCIT treatment is administered for $\sim 3$ years and includes two phases: Dose accumulation and dose maintenance. SIT can be divided into conventional immunotherapy and cluster immunotherapy during the dose accumulation phase. In conventional immunotherapy, the dose accumulation phase consists of injections of increasing doses of the allergen once a week, with the maintenance dose achieved after $\sim 15$ weeks. However, during the dose accumulation phase of cluster immunotherapy, the allergen is injected 2-3 times in 1 day each week, 30 min apart, thus the maintenance dose can be achieved within $\sim 6$ weeks. Therefore, the frequency of visits can be reduced and the maintenance dose is achieved quickly when using the cluster immunotherapy regimen.

A limited number of studies have compared the efficacy and safety of conventional immunotherapy with cluster immunotherapy. Therefore, the present study aimed to compare the efficacy and safety of conventional and cluster immunotherapy regimens in 60 patients with AR.

\section{Materials and methods}

Subjects. Patients were treated at the Desensitization Treatment Center in the Department of Otolaryngology at the Second Affiliated Hospital \& Yuying Children's Hospital of Wenzhou Medical University (Wenzhou, China) between January 2009 and December 2012. Diagnostic criteria for AR was set by the Otolaryngology branch of the Chinese Medical Association. The inclusion and exclusion criteria for patients enrolled in the standard immunotherapy against dust mite were as follows:

Inclusion criteria: i) Aged 5-35 years; ii) diagnosed with AR or AR complicated by asthma, forced expiratory volume in one second: $\geq 70 \%$ of normally predicted value; iii) Skin Prick Test (ALK-Abelló, Hørsholm, Denmark) of the patient 


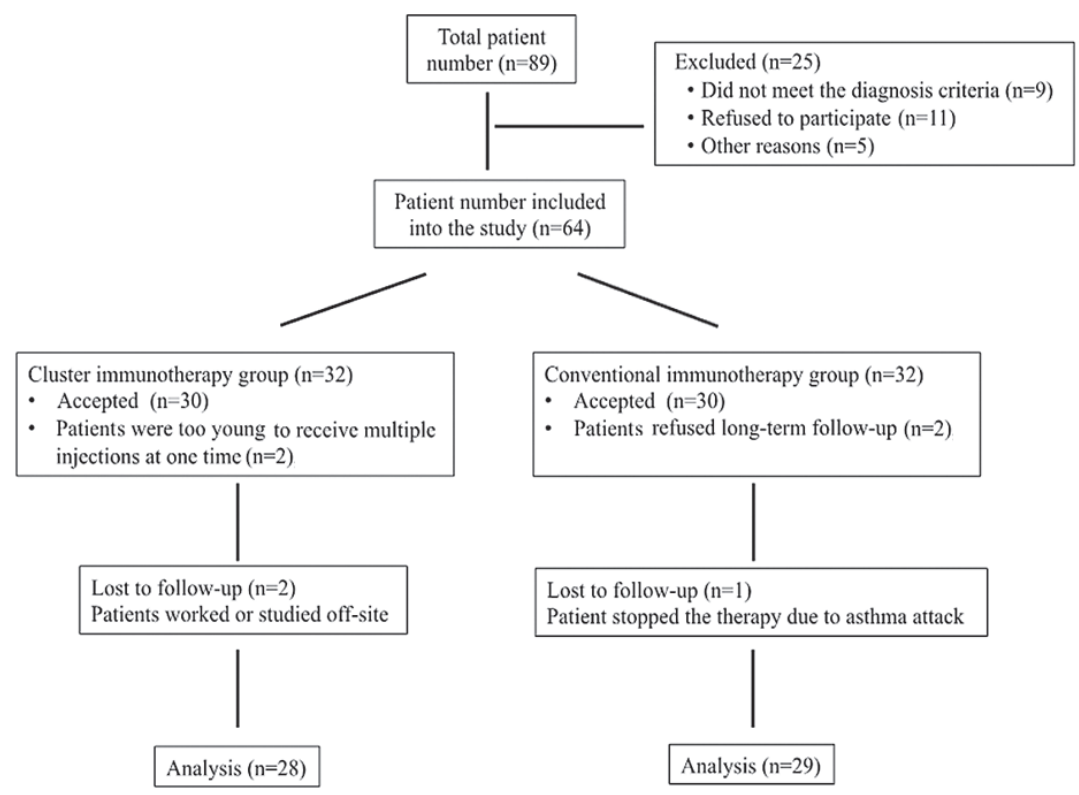

Figure 1. Flow diagram of the specific immunotherapy administered to patients with allergic rhinitis in the present study.

should be positive for the mite allergen (including house dust mites and dust mites) with a skin test index $\geq 0.5(++)$ and the concentration of specific IgE to serum dust mites in the patient should be $>0.7 \mathrm{KU} / \mathrm{L}$. All patients who met all three criteria were enrolled into the present study.

Exclusion criteria included: i) immune diseases other than an allergy, chronic heart and lung disease or renal dysfunction; ii) severe immune complications or malignant tumor; iii) adrenaline contraindication; iv) patients who lacked collaboration or exhibited a severe mental disorder.

Following exclusion, 60 patients with AR against dust mites met the inclusion criteria, including 46 men and 14 women, aged 5-32.5 years, and nine of these cases also suffered from asthma. The present study was conducted as an open and comparative trial, with the treatment regimen chosen by each patient, with informed consent. A flow chart of the study is shown in Fig. 1.

Follow-up data was obtained in 28 cases in the cluster immunotherapy group, and 29 cases in the conventional immunotherapy group. These 57 patients are described in Table I, demonstrating that no differences in gender or age were detected between the two groups. Complaints from patients included nasal itching, sneezing and clear nasal discharge. Skin Prick Test was positive for the Dermatophagoides pteronyssinus dust mite allergen in all cases. Other inhaled allergens were all less than ++ , including spring pollen, autumn pollen, polyvalent mold, polyvalent animal hair, polyvalent feather and cockroach. Specific IgE to serum dust mites were tested and all patients exhibited concentrations $>0.7 \mathrm{KU} / \mathrm{L}$ (UniCAP100; Phadia AB, Uppsala, Sweden). Patients were diagnosed with asthma after demonstrating airflow obstruction (Micro Medical Ltd, Chatham, UK), with a peak expiratory flow of $>70 \%$ demonstrated in all patients.

Standard vaccine immunotherapy against dust mite allergen. All patients received standard vaccine immunotherapy against dust mite allergen (Alutard ${ }^{\circledR}$ SQ; ALK-Abelló). Each treatment course included a dose accumulation phase and a dose maintenance phase. During the dose accumulation phase, the initial dose in the conventional group was $20 \mathrm{SQ}-\mathrm{U}$ via one injection a week, which was escalated weekly until a maintenance dose of 100,000 SQ-U was achieved (15 weeks). This dose remained constant and the injection interval was extended to 6-8 weeks. In the cluster group, the initial dose was $10 \mathrm{SQ}-\mathrm{U}$ with 2-3 injections performed $30 \mathrm{~min}$ apart weekly. Patients were observed for $30 \mathrm{~min}$ and adverse reactions following injection were recorded. The maintenance dose of 100,000 SQ-U was reached after $\sim 6$ weeks, after which the dose remained constant and the injection interval was extended to 6-8 weeks (Table II).

Efficacy evaluation. Nasal mucosa scores were recorded at each visit, according to symptom and sign grading standards of allergic nasal mucosa inflammation. Five symptoms were used in symptom grading: Nasal itching, sneezing, nasal discharge flow, nasal obstruction and itching of the eye. Each symptom was graded as follows: 0, no symptoms; 1 , mild symptoms (3-5 sneezes every day); 2, moderate symptoms (6-10 sneezes every day); and 3 , severe symptoms ( $>10$ sneezes every day). The sign score was graded on a scale of $0-3$ : 0 , normal; 1 , mild swelling of the inferior nasal concha, nasal septum and middle nasal concha are visible; 2, inferior nasal concha is near the nasal septum (or basis nasi), with a visible gap between inferior nasal concha and basis nasi (or nasal septum); 3, inferior nasal concha is near the basis nasi or nasal septum, middle nasal concha is not visible, or a mucosal polyp is visible in the middle nasal concha. Total score was calculated by totaling the symptom and sign scores. Efficacy evaluation was scored according to the standard efficacy measures of AR outlined by the 1997 Haikou conference (4): Improvement percentage was (total score before treatment - total score after treatment)/total score before treatment $x 100 \%$. A value of $\geq 51 \%$ demonstrated an obvious effect, $21-50 \%$ indicated effective, whereas $\leq 20 \%$ was considered to indicate no effect. Efficacy was evaluated 
Table I. Clinical characteristics of patients with allergic rhinitis (AR) who completed follow-up.

\begin{tabular}{lcc}
\hline Characteristic & Cluster group & Conventional group \\
\hline Number of cases & 28 & 29 \\
Medical history of AR, years $^{\mathrm{a}}$ & $5(1.5-10)$ & $4(2.5-8.5)$ \\
Age, years $^{\mathrm{a}}$ & $23.5(8.5-30)$ & $22.0(5.5-32.5)$ \\
Sex, M/F & $22 / 6$ & $21 / 8$ \\
Asthma & 5 & 4 \\
Total score of signs and symptoms & \\
Positive for Dermatophagoides pteronyssinus allergen & $12.1(4-19)$ & $12.7(5-18.5)$ \\
& $++: 7$ cases & $++: 6$ cases \\
& $+++: 8$ cases & +++9 cases \\
\end{tabular}

${ }^{\text {a }}$ Data are presented as the median $\left(25-75^{\text {th }}\right.$ percentiles $)$.

Table II. Immunotherapy regimens for patients enrolled in the cluster and conventional immunotherapy groups.

\begin{tabular}{crrrrr}
\hline & \multicolumn{2}{c}{ Cluster } & & \multicolumn{2}{c}{ Conventional } \\
\cline { 2 - 3 } Week & $\begin{array}{c}\text { Injection } \\
\text { (visit) }\end{array}$ & $\begin{array}{c}\text { Dose } \\
\text { (SQ-U) }\end{array}$ & & $\begin{array}{c}\text { Injection } \\
\text { (visit) }\end{array}$ & $\begin{array}{r}\text { Dose } \\
\text { (SQ-U) }\end{array}$ \\
\hline 0 & $1(1)$ & 10 & $1(1)$ & 20 \\
1 & $2(1)$ & 100 & & \\
& $3(1)$ & 1,000 & & \\
& $4(2)$ & 2,000 & $2(2)$ & \\
2 & $5(2)$ & 4,000 & & \\
& $6(3)$ & 5,000 & $3(3)$ & \\
& $7(3)$ & 10,000 & & \\
3 & $8(4)$ & 10,000 & & $4(4)$ & 200 \\
& $9(4)$ & 20,000 & & \\
4 & $10(5)$ & 20,000 & $5(5)$ & 400 \\
& $11(5)$ & 40,000 & & \\
5 & $12(6)$ & 40,000 & $6(6)$ & 800 \\
& $13(6)$ & 60,000 & & \\
6 & $14(7)$ & 100,000 & $7(7)$ & 2,000 \\
7 & - & - & $8(8)$ & 4,000 \\
8 & $15(8)$ & 100,000 & $9(9)$ & 8,000 \\
9 & - & - & $10(10)$ & 10,000 \\
10 & - & - & $11(11)$ & 20,000 \\
11 & - & - & $12(12)$ & 40,000 \\
12 & $16(9)$ & 100,000 & $13(13)$ & 60,000 \\
13 & - & - & $14(14)$ & 80,000 \\
14 & - & - & $15(15)$ & 100,000 \\
\hline & & & & &
\end{tabular}

- indicates no visit and injection.

according to the symptoms and signs prior to standard therapy, 6 weeks post-treatment, and 1 year post-treatment.

Safety evaluation. Patients remained in the clinic for at least $30 \mathrm{~min}$ following each injection, and adverse reactions were recorded during this period. Adverse reactions included local reactions (wheal, calluses, itching, blush or papule), and systemic adverse reactions, which included generalized urticaria, sneezing, nasal discharge flow, itching of the eye, rhinocnesmus, asthma, cough, drop in blood pressure, shock or loss of consciousness. Systemic adverse reactions were evaluated according to the grading system recommended by European Academy of Allergy and Clinical Immunology: Grade 0 , no symptoms or non-specific symptoms; grade I (mild), local urticaria, rhinitis or mild asthma [peak expiratory flow rate (PEFR) falls $<20 \%$ from baseline]; grade II (moderate), generalized urticaria appeared slowly ( $>15 \mathrm{~mm}$ ) and/or moderate asthma (PEFR falls by $<40 \%$ ); grade III (severe), generalized urticaria appeared rapidly $(<15 \mathrm{~mm})$, angioedema or severe asthma (PEFR falls by $>40 \%$ ); and grade IV (allergic shock), pruritus, flush, erythema, generalized urticaria, sneezing (angioedema), asthma or a sharp decrease in blood pressure.

Statistical analysis. SPSS 17.0 statistical software (SPSS, Inc., Chicago, IL, USA) was used to perform statistical analysis with $\chi^{2}$ tests. $\mathrm{P}<0.05$ was considered to indicate a statistically significant difference.

\section{Results}

Patient characteristics. Among the 60 patients enrolled in the present study, 57 completed follow-up: cluster immunotherapy group $(n=28)$ and conventional immunotherapy group $(n=29)$. Two out of the three cases lost to follow-up were from the cluster immunotherapy group, and the remaining case was from the conventional group. Four patients experienced adverse generalized reactions several times during the treatment and their dose timetables were adjusted to ensure their safety. All four patients were treated with conventional immunotherapy and the dose accumulation phase remained unfinished after 1 year of treatment, with a maintenance dose at 80,000 SQ-U.

Clinical efficacy of the two regimens. Following 6 weeks of treatment, the cluster regimen exhibited a greater effect than the conventional regimen $\left(\chi^{2}=7.617\right.$; $P<0.01$; Table III; Fig. 2). 
Table III. Comparison of clinical efficacy after 6 weeks of treatment.

\begin{tabular}{lccccc}
\hline Group & Obviously effective & Effective & No effect & Total & Efficiency $(\%)$ \\
\hline Cluster & 14 & 10 & 4 & 28 & $85.71^{\text {a }}$ \\
Conventional & 8 & 7 & 14 & 18 & 51.72 \\
Total & 22 & 17 & 57 & 68.42 \\
\hline
\end{tabular}

${ }^{\mathrm{a}} \mathrm{P}<0.01$ vs. the conventional group.

Table IV. Comparison of clinical efficacy after 1 year of treatment.

\begin{tabular}{lccccc}
\hline Group & Obviously effective & Effective & No effect & Total & Efficiency $(\%)$ \\
\hline Cluster & 13 & 9 & 6 & 28 & $78.57^{\text {a }}$ \\
Conventional & 12 & 10 & 7 & 29 & 75.86 \\
Total & 25 & 19 & 13 & 57 & 77.19 \\
\hline
\end{tabular}

${ }^{\mathrm{a}} \mathrm{P}>0.05$ vs. the conventional group.

However, after 1 year of therapy, the two treatment groups exhibited equivalent levels of efficacy $\left(\chi^{2}=0.059 ; \mathrm{P}>0.05\right.$; Table IV; Fig. 1). This finding is likely to be due to the dose being much higher by the sixth week in the cluster group, as compared with the conventional group, and this demonstrates that the cluster regimen is able to improve symptoms faster and earlier in the treatment regimen. These results demonstrated that the effective rate after 1 year of treatment was less than that after 6 weeks in the cluster group. Many patients exhibited an improvement in symptoms; however, this effect was reduced with more injections. Conversely, the effective rate in the conventional group increased from $51.72 \%$ after 6 weeks of treatment to $75.86 \%$ after 1 year of treatment (Tables III and IV). Local adverse reactions were common in both groups (Table V). Symptoms all regressed. There was no difference in the incidence of adverse reactions during the dose accumulation or maintenance phases between the two groups $\left(\chi^{2}=0.233\right.$ and $\chi^{2}=0.017$, respectively; $\left.P>0.05\right)$.

Systemic adverse reactions during the dose accumulation phase. Table VI demonstrates that a similar frequency of systemic adverse reactions during the dose accumulation phase of each treatment was observed in both groups $\left(\chi^{2}=0.01\right.$; $\mathrm{P}>0.05)$. All adverse reactions occurred at least $30 \mathrm{~min}$ after injection. Reactions of grade I regressed rapidly following drug treatment (aerosol inhalation of Combivent ${ }^{\circledR}$ and Pulmicort ${ }^{\circledR}$ ). Reactions of grade II regressed rapidly following drug treatment with aerosol inhalation of Combivent ${ }^{\circledR}$ and Pulmicort ${ }^{\circledR}$, and oral loratadine. Three cases regressed after subcutaneous injection of epinephrine. Hospitalization was not required in any of the cases.

Systemic adverse reactions during the dose maintenance phase. Table VII shows the incidence of systemic adverse reactions during the dose maintenance stage of each treatment group. Two patients in the cluster immunotherapy group

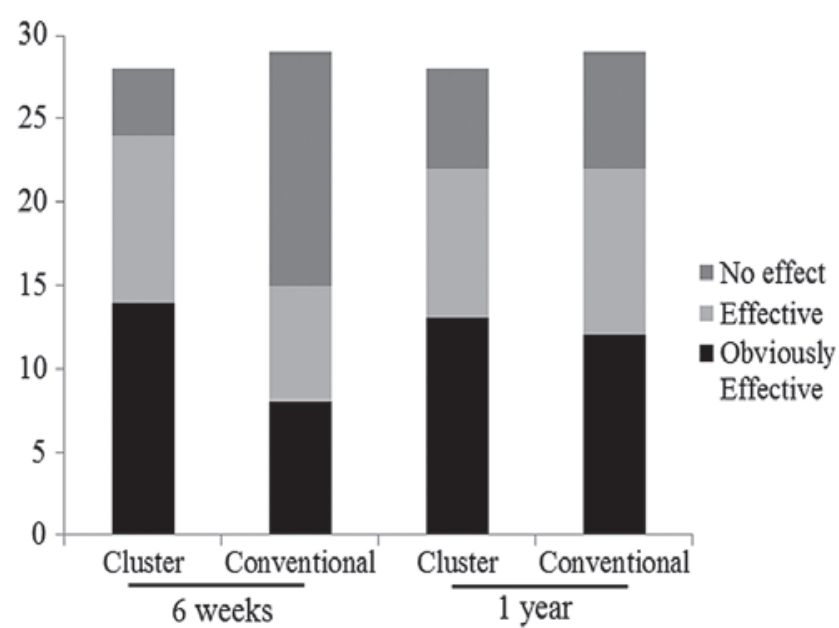

Figure 2. Clinical efficacy 6 weeks and 1 year post-treatment.

demonstrated four incidences of adverse reactions in total, of which two were rated as grade I and two were rated as grade II. The incidence of adverse reaction was $1.58 \%$. In the cluster immunotherapy group, two patients demonstrated three incidences of adverse reactions in total. Two were rated as grade I and the other one was rated as grade II. The incidence was $1.56 \%$, which was not significantly different from that of the cluster group $\left(\chi^{2}=0.02 ; \mathrm{P}>0.05\right)$.

\section{Discussion}

Currently in China, standard allergen vaccination against dust mites is used as the conventional dosing regimen of immunotherapy and cluster therapy is rarely used (5). During treatment, particularly during the dose accumulation phase, patients being treated with the conventional dosing regimen complete more visits, and the dose may require adjustment if patients suffer from infections during treatment. Therefore, the 
Table V. Local adverse reactions observed during the respective dose accumulation stages of the therapy regimen.

\begin{tabular}{|c|c|c|c|c|c|c|c|}
\hline \multirow[b]{2}{*}{ Group } & \multirow[b]{2}{*}{ Case } & \multicolumn{3}{|c|}{ Injection number } & \multicolumn{3}{|c|}{ Incidence of adverse reaction } \\
\hline & & Accumulation & Maintenance & Total & Accumulation & Maintenance & Total \\
\hline Cluster & 28 & 394 & 222 & 616 & $11(2.79)$ & $12(5.41)$ & $23(3.73)$ \\
\hline Conventional & 29 & 445 & 193 & 638 & $15(3.37)$ & $11(5.70)$ & $26(4.08)$ \\
\hline Total & 57 & 839 & 415 & 1,254 & & & \\
\hline
\end{tabular}

Table VI. Systemic adverse reactions during the respective dose accumulation stages of the therapy regimen.

\begin{tabular}{lcccccr}
\hline \multirow{2}{*}{ Group } & & & & \multicolumn{2}{c}{ Incidence of adverse reaction (\%) } \\
\cline { 5 - 7 } & Cases & Cases with reactions (\%) & Times of injection & Grade I & Grade II & Total \\
\hline Cluster & 28 & $3(10.71)$ & 394 & $3(0.77)$ & $2(0.50)$ & $5(1.27)$ \\
Conventional & 29 & $4(13.79)$ & 445 & $4(0.90)$ & $2(0.45)$ & $6(1.35)$ \\
\hline
\end{tabular}

Table VII. Systemic adverse reactions during the respective dose maintenance stages of the therapy regimen.

\begin{tabular}{lcccccr}
\hline \multirow{2}{*}{ Group } & & & & \multicolumn{2}{c}{ Incidence of adverse reaction (\%) } \\
\cline { 5 - 7 } & Cases & Cases with reactions (\%) & Times of injection & Grade I & Grade II & Total \\
\hline Cluster & 28 & $2(7.14)$ & 252 & $2(0.79)$ & $2(0.79)$ & $4(1.58)$ \\
Conventional & 29 & $4(6.90)$ & 193 & $4(1.04)$ & $1(0.52)$ & $3(1.56)$ \\
\hline
\end{tabular}

dose accumulation phase is long, which is usually the reason why patients do not want to be treated with immunotherapy or are lost to follow-up. However, cluster therapy can greatly reduce the duration of the dose accumulation phase, ensuring that patients enter the dose maintenance phase quickly. This improves the efficiency of SCIT and thus improves compliance, which is important for children in school, who are able to complete the cluster therapy over summer or winter vacation without affecting school attendance. In the present study, two patients were lost to follow-up in the cluster immunotherapy group due to long-distance work or study, whereas one patient terminated the treatment due to an asthma attack in the conventional immunotherapy group. Using the cluster SCIT therapy dosing regimen described herein, patients reached the maintenance dose phase in 6 weeks, reducing the dose accumulation phase by $>60 \%$ compared with conventional group. All cases reached the dose maintenance phase successfully; no severe complications or hospitalizations occurred. This demonstrated the advantages of cluster dose accumulation therapy in treating AR using the standard allergen vaccine against dust mites (Alutard ${ }^{\circledR} \mathrm{SQ}$ ) (6).

Immunotherapy is only defined as clinically significant when a patient's symptom grade is reduced by $>30 \%$. According to this criterion, in 57 patients with AR, after 1 year's treatment the total effective rate was $77.2 \%$, which is similar to the $75.0 \%$ reported by Chang and Hong (7) when treating AR through immunotherapy. The majority of patients are allergic to both house dust mites and dust mites, since they possess similar allergenicity, and this may be one reason for the good effect demonstrated in the present patients. The two treatment regimens exhibited substantial effects after 6 weeks, with the cluster group demonstrating a more obvious effect than the conventional group. Although the exact mechanism of this effect remains unknown, the treatment effect after 1 year demonstrated in the present study was satisfying and clinically meaningful. The WHO recommends that immunotherapy should last for at least 3 years; however, the present patients were only observed for 1 year. Therefore, further clinical observation is required to assess the long-term effects of these two treatment regimens.

Local or systemic adverse reactions may occur during immunotherapy, which is associated with each patient's sensitivity to allergens, the dose of the vaccine, duration and route of injection, the patient's condition, whether a delayed absorption carrier is used in the vaccine, and potentially other undiscovered reasons $(8,9)$. Following 1 year of observation of the present 57 patients, the incidence of local adverse reactions was high (29.70 and $39.33 \%$ ) in the two groups during the dose accumulation phase; after 1 year of treatment, the incidence peaked at 43.99 and $45.61 \%$. Local adverse reactions may occur more frequently during the dose maintenance phase. A previous study has demonstrated that during treatment of persistent AR using the standard allergen vaccine against dust mites (Alutard ${ }^{\circledR} \mathrm{SQ}$ ), most local adverse reactions occurred when the dose was $>4,000 \mathrm{SQ}-\mathrm{U}$ (10). This indicates that there may be a correlation between local adverse reactions and the 
dose of vaccine during immunotherapy. However, local adverse reactions regress without any treatment; therefore, there is no difference in safety between the two groups. According to previous research (5), 5.9\% of patients experience a systemic adverse reaction when treated with cluster immunotherapy ( $0.75 \%$ of all injections). However, the present study found that after 1 year of treatment, the incidence of systemic adverse reactions in the cluster immunotherapy group was $17.86 \%$ of patients and $1.46 \%$ of all injections. The increased percentage of systemic adverse reactions in the present patients, as compared with previous studies, may be due to the fact that some patients also had asthma in addition to the AR.

During the dose accumulation phase, systemic adverse reactions occurred in four cases $(13.79 \%)$ with six incidences $(1.35 \%)$ in the conventional therapy group, and three cases $(10.71 \%)$ with five incidences $(1.27 \%)$ in the cluster immunotherapy group. Systemic adverse reactions after 1 year of immunotherapy were evaluated according to the grading system recommended by European Academy of Allergy and Clinical Immunology. Reactions regressed rapidly following drug treatment, suggesting that cluster immunotherapy is safe for AR. A double-blind study conducted by Tabar et al (11) contrasted the safety of conventional and cluster immunotherapy, and demonstrated no statistical difference. Although no severe systemic adverse reactions occurred in the present patients, severe adverse reactions that are life-threatening, such as severe asthma and allergic shock, may happen during immunotherapy. Therefore, allergen specific immunotherapy should be performed by medical professionals in medical institutions with rescue facilities.

The results of the present study suggested that cluster immunotherapy is able to improve symptoms in patients with AR caused by dust mites, with no increase in adverse reactions. Therefore, cluster immunotherapy may be a rapid, effective, safe treatment for moderate to severe persistent AR.

\section{References}

1. Passalacqua G and Durham SR; Global Allergy and Asthma European Network: Allergic rhinitis and its impact on asthma update: Allergen immunotherapy. J Allergy Clin Immunol 119: 881-891, 2007.

2. Bousquet J, Khaltaev N,Cruz AA, Denburg J,Fokkens WJ, Togias A, Zuberbier T, Baena-Cagnani CE, Canonica GW, van Weel C, et al; World Health Organization; GA(2)LEN; AllerGen: Allergic Rhinitis and its Impact on Asthma (ARIA) 2008 update (in collaboration with the World Health Organization, GA(2)LEN and AllerGen). Allergy 63 (Suppl 86): 8-160, 2008.

3. Brozek JL, Baena-Cagnani CE, Bonini S,Canonica GW, Rasi G, van Wijk RG, Zuberbier T, Guyatt G, Bousquet J and Schünemann HJ: Methodology for development of the Allergic Rhinitis and its Impact on Asthma guideline 2008 update. Allergy 63: 38-46, 2008.

4. Diagnostic criteria and curative effect evaluation standard of allergic rhinitis (1997 revision, Haikou). Zhong Hua Er Bi Yan Hou Ke Za Zhi: 6-7, 1998 (In Chinese).

5. Zhang L, Wang CS, Wang XD, Zhao Y, Liu JH, Zhang W, Zhou B and Han DM: Efficacy and safety of cluster immunotherapy for 154 patients with allergic rhinitis. Zhonghua Er Bi Yan Hou Tou Jing Wai Ke Za Zhi 43: 187-191, 2008 (In Chinese).

6. Wang CS, Zhang W, Wang XD, Xi L, Ouyang YH, Zhao Y and Zhang L: Comparative study on cluster and conventional immunotherapy with Dermatophagoides pteronyssinus in patients with allergic rhinitis. Zhonghua Er Bi Yan Hou Tou Jing Wai Ke Za Zhi 46: 981-985, 2011 (In Chinese).

7. Chang J and Hong CS: The effect of immunotherapy on nonspecific bronchial hyperresponsiveness in bronchial asthma and allergic rhinitis. Yonsei Med J 42: 106-113, 2001.

8. Alvarez-Cuesta E, Bousquet J, Canonica GW, Durham SR, Malling HJ and Valovirta E; EAACI, Immunotherapy Task Force: Standards for practical allergen-specific immunotherapy. Allergy 61 (Suppl 82): 1-20, 2006.

9. Nelson HS: Allergen immunotherapy: Where is it now? J Allergy Clin Immunol 119: 769-779, 2007.

10. Han H, Chen SH and Qiu QH: Curative effect and safety evaluation of Alutard dust mite vaccine for the treatment of allergic rhinitis. Guangdong Yi Xue 28: 991-992, 2007 (In Chinese).

11. Tabar AI, Echechipía S, García BE, Olaguibel JM, Lizaso MT, Gómez B, Aldunate MT, Martin S and Marcotegui F: Double-blind comparative study of cluster and conventional immunotherapy schedules with Dermatophagoides pteronyssinus. J Allergy Clin Immunol 116: 109-118, 2005. 